\title{
ACCURATE BINARY MASS DETERMINATIONS: GOALS, LIMITATIONS, AND PROSPECTS
}

\author{
J. ANDERSEN \\ Niels Bohr Institute for Astronomy, Physics, and Geophysics \\ Astronomical Observatory \\ Brorfeldevej 23 \\ DK - 4340 Tollese, Denmark
}

\begin{abstract}
The state of the art in accurate mass determination for binary stars is reviewed, and the angular sizes and their errors are computed for a typical system from the existing high-precision sample. It appears that sub- $\mu$ as (microarcsecond) absolute astrometry will be needed in order to improve the accuracy substantially by astrometry alone. The types of system, and the kinds of data, where precision astrometry appears most promising are outlined. Finally, astrophysical applications of such accurate stellar masses, and the auxiliary data required in them, are briefly reviewed.
\end{abstract}

\section{Introduction}

When this contribution was first suggested, the proposed subject was the improvement in state-of-the-art mass determinations for eclipsing binary stars that could be expected to result from new parallaxes with errors in the 10-100 $\mu$ as range. The ultrashort summary reply, "Not much", is clearly not very useful unless backed by an explanation of the state of the art and the possibilities and limitations in improving it. Also, while determining precise stellar masses may seem to be a worthy goal in itself, a reasonably focused idea of the precise astrophysical applications of the data is a useful basis in such work.

Hence, the following will review the state of the art in mass determinations for (doublelined) eclipsing binaries, translate the results into visual binary language for comparison with the projected astrometric capabilities, and outline the practical possibilities and likely results. As will appear, very useful data can indeed result, if perhaps not always primarily on stellar masses per se. Finally, to place these data in a specific astrophysical context, I shall outline some of the uses of precise stellar masses that motivate their determination, and the supporting data which are also needed in such applications.

\section{The State of the Art in Stellar Masses}

It is elementary textbook material that in eclipsing binaries in which the lines of both stars are visible in the combined spectrum, the individual masses are determined from the two orbital velocities (radial-velocity amplitudes), combined with the orbital inclination as determined from the light-curve analysis. In the process, the absolute radii are also found, and individual luminosities are computed from these radii combined with effective temperatures derived from suitable calibrated (and reddening-corrected) colour index measurements. 
These results are entirely independent of the distance of the system, which then follows from the apparent magnitude, reddening-corrected as appropriate.

In astrometric binaries, the astrometry yields the orbital semi-major axis $a$ (and $e, \omega$ ) and inclination of the orbit, plus the parallax, hence the distance. From these, the sum of the masses is obtained, as well as the individual luminosities if $\Delta m$ is well determined. Determination of individual masses requires that either absolute astrometric orbits or a double-lined spectroscopic orbit is available. Radii follow from the luminosities when effective temperatures have been determined from colour indices as above.

For the basic reasons well explained by Popper (1980), the most accurate mass determinations are possible in eclipsing binaries. Published values of individual masses and radii of the components show errors down to $\sim 0.5 \%$. The available sample of accurate binary mass (and radius) determinations, defined as having errors of no more than $2 \%$ in both masses and radii, comprises about 45 systems, all double-lined eclipsing binaries. These systems are listed and discussed by Andersen (1991), who also addresses in considerable detail the precautions necessary in the selection of systems as well as in the observations and analysis in order to reach this level of accuracy. Hence, that discussion will not be repeated here.

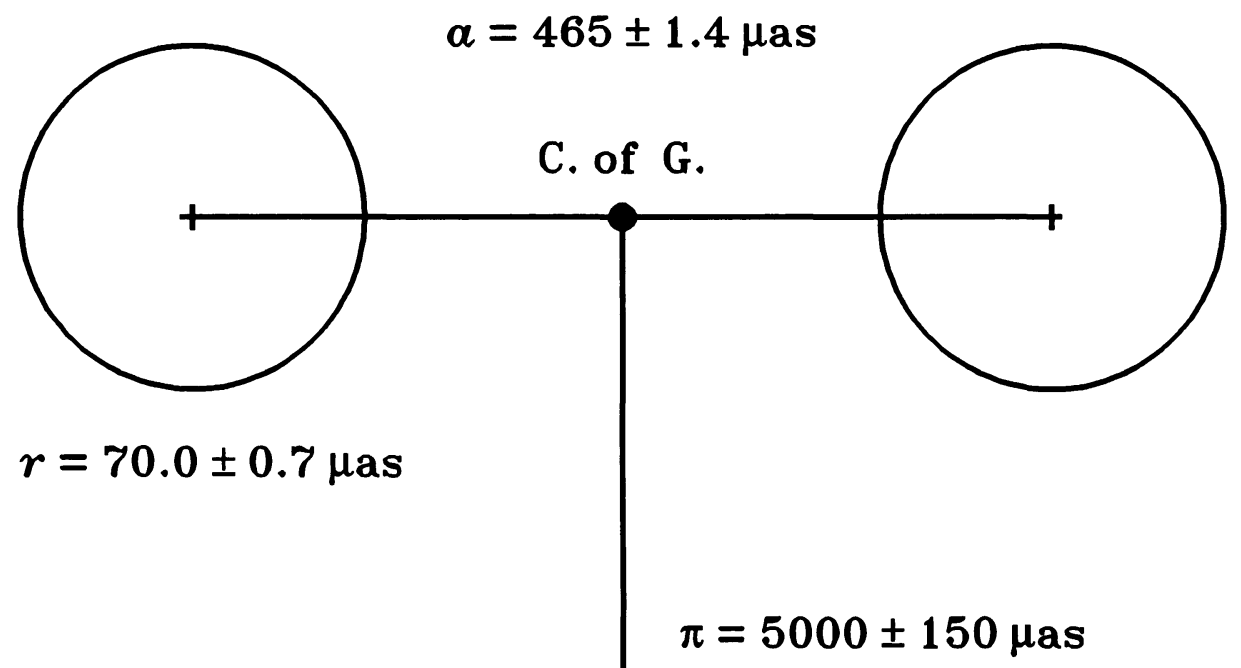

Figure 1. Angular dimensions of the typical binary system from Table 1 when placed at the typical distance of $200 \mathrm{pc}$. 
From the list of stellar properties in Table 1 of Andersen (1991), the characteristics of a typical system have been extracted; they are summarised in Table 1 below:

TABLE 1. Typical properties of a well-studied binary system. The full parameter ranges observed in the sample are also given for comparison.

\begin{tabular}{lrcr}
\hline Parameter & Value & Range & Accuracy \\
\hline Mass & $2 M_{\odot}$ & $0.6-23 M_{\odot}$ & $1 \%$ \\
Radius & $3 R_{\odot}$ & $0.6-9 R_{\odot}$ & $1 \%$ \\
Luminosity & $20 L_{\odot}$ & $0.1-10^{5} L_{\odot}$ & $10 \%$ \\
Orbital radius & $20 R_{\odot}$ & $4-120 R_{\odot}$ & $0.3 \%$ \\
Distance & $200 \mathrm{pc}$ & $15-2200 \mathrm{pc}$ & $3 \%$ \\
\hline
\end{tabular}

Taking the typical system described in Table 1 and placing it at the distance indicated leads to the set of angular separations and diameters shown, with their errors, in Fig. 1.

It is obvious that sub- $\mu$ as accuracy is needed to improve the determination of the intrinsic stellar parameters mass and radius substantially from astrometry alone: Assuming that the error of the parallax were only $25 \mu$ as, and the individual semi-major axes known without error (e.g. from ultra-precise spectroscopic orbits), the mass errors would still amount to $1.5 \%$, not yet competitive with the best existing spectroscopic determinations.

Hence, the initial answer to the question how sub-milliarcsecond astrometry could improve the precision of state-of-the-art stellar mass determinations does seem to be: "Not much".

\section{The scope for astrometric contributions}

Two comments are needed to qualify the negative statement just made. First, it only applies to the types of star which are found in suitable eclipsing binary systems that have also been adequately studied, i.e., with only a couple of exceptions, main-sequence stars of Population I in the range G5 - $08\left(0.6-23 M_{\odot}\right)$. Stars at the lowest and highest ends of the main sequence, and stars evolved to the giant stage or beyond, are either not generally found in eclipsing binary systems, or their properties (spectra, light curves, brightness) are not suitable for accurate analysis of the kind performed for the well-behaved main-sequence stars referred to above. For such stars, masses of lower accuracy may still be valuable, and/or the masses may be of interest even without the accompanying data discussed below.

It follows that astrometric studies can be profitably concentrated on systems that:

- are much closer than $200 \mathrm{pc}$, so larger errors can be tolerated, or

- have no measurable spectral lines in at least one component, or

- are not eclipsing, but have

either: absolute astrometric orbits of both stars,

or: $\quad$ spectroscopic orbits of both stars.

Stellar types for which such determinations would be of particular interest include, on the main sequence, $\mathrm{O}$ stars at the high end and $\mathrm{K}-\mathrm{M}$ dwarfs and brown dwarfs at the low end; evolved stars such as giants and supergiants, hot subdwarfs, white dwarfs, or nuclei of 
planetary nebulae; and metal-poor Population II stars of all types.

Second, by improving the parallax error to $0.5 \%$ (Fig. 1) something $i s$, of course, gained, because the error in the individual luminosities is reduced from $\sim 10 \%$ to $\sim 1 \%$. It would appear to be of little specific value to do this for binary rather than single stars, though, unless some observational parameter were known particularly well for the binary stars (see below). In eclipsing binary stars, however, a further valuable result follows since the surface areas of the stars are known: The effective temperature may be determined.

From the well-known relation:

$$
L=4 \pi R^{2} \cdot \sigma T_{e}^{4}
$$

it is seen that if both $L$ and $R$ are known to $0.5-1 \%$, we can derive $T_{e}$ to an accuracy of about $0.5 \%$, much better than with most current temperature calibrations. A recent example of this technique is given by Nordström \& Johansen (1994) for the bright, nearby system $\beta$ Aurigae. Again, application of this knowledge to other stars requires that very accurate observational parameters are available for their characterisation.

\section{Why are accurate masses useful at all, and under what conditions?}

Even the world's most accurate mass determination for a star is without interest outside the Guinness Book of Records unless it can be used to address some astrophysical question of wider implications. So what useful applications can one envisage for a sample of accurate empirical determinations of stellar masses (say, $1 \%$ or better)? The three main applications of which the author is aware (see further discussion in Andersen 1991) are:

- Predicting masses (and radii) for single stars from observed parameters,

- Improving "the" mass-luminosity relation, and

- Testing stellar evolution computations.

However, in all three cases, the factor limiting the accuracy with which the key relations can be defined, or theory and observation be compared, is not the observational error of the actual masses once it is below the $1 \%$ level. Specifically, in each of the above cases:

- As shown by the accurate data compiled by Andersen (1991), observed masses and radii for main-sequence stars with a given spectral type or colour, however accurately determined, span a range of $\sim 30 \%$ in $M$ and a factor 2 in $R$, due primarily to differences in evolution even within the main-sequence band. The best measure of post-ZAMS evolution is $\log g$ as determined fundamentally and with superior precision $(0.01$ dex or better, $\sim 5 \%$ of the width of the main sequence) in eclipsing binaries, directly from $M$ and $R$. Taking evolution into account allows to reproduce the observed $M$ and $R$ within $\sim 5 \%$; the excess scatter relative to the observational error of $1 \%$ is probably due to differences in chemical composition (metal abundance). Hence, knowing the mass of a star to $1 \%$ is not really useful unless $\log g$ and $[\mathrm{Fe} / \mathrm{H}]$ are also known to matching accuracy.

- Similarly, the scatter of the observed points around a mean mass-luminosity relation defined from the same sample of stars cannot be explained only by observational errors in the masses (or luminosities); see Fig. 2. Most of the scatter is due to real differences in, again, evolution above the ZAMS but within the main sequence, and in metal abundance from star to star. Improving only the precision of the masses (or even $M$ and $L$ ) would not lead to a better-defined mean relation. 


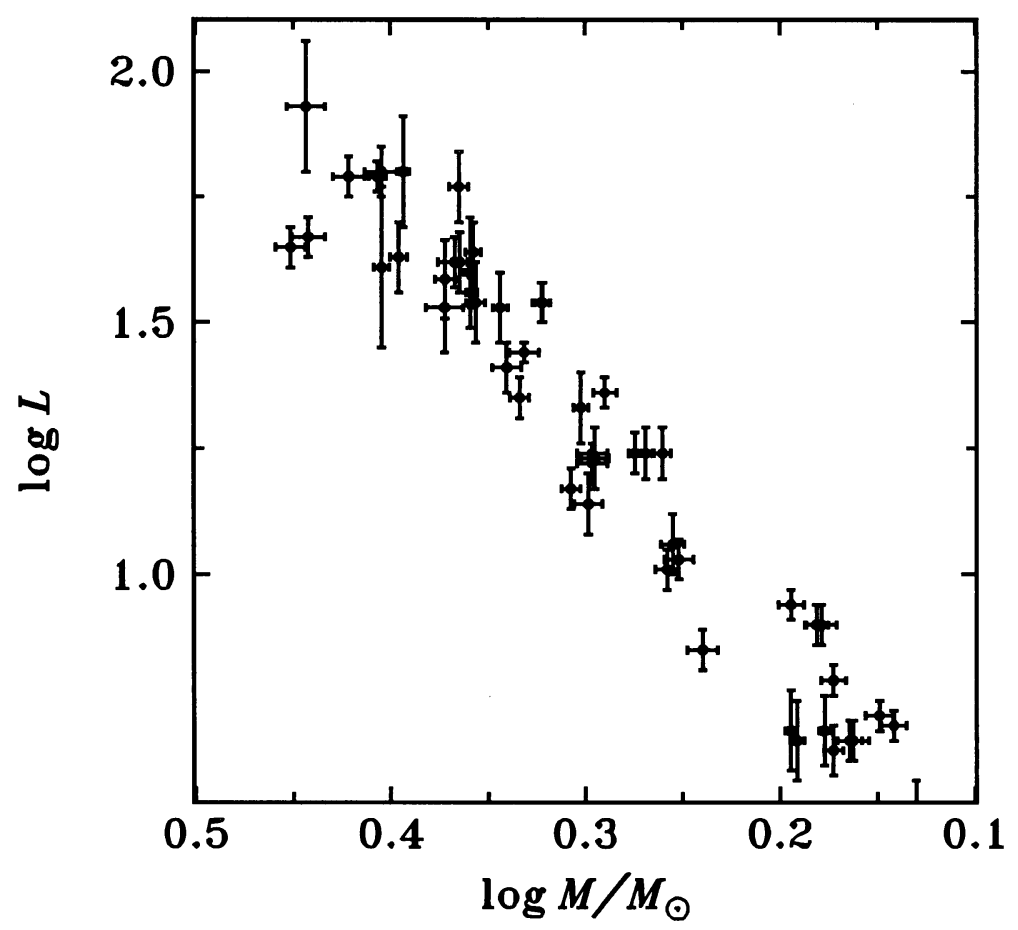

Figure 2. Zoom-in on a section of the mass-luminosity relation as defined by data from Table 1 of Andersen (1991). Note that the scatter considerably exceeds the observational errors.

- Precise observational tests of the predictions of stellar evolution theory is one of the most demanding applications of empirical data on stellar masses and radii. Basically, one evolves a model of the mass and composition of one of the binary components to match its observed radius and luminosity, fixing such model parameters as mixing length and helium abundance from a model of the present Sun. The test then consists of computing a model for the mass of the other star, but with the age and all other parameters unchanged, and verifying whether it fits all observed properties of that star as well, within the errors. Yet, while initial mass is the most important property of a stellar model, the metal abundance turns out in fact to be the limiting factor in fitting models to the observations as illustrated, e.g. by Andersen et al. (1989) who actually determined [Fe/H] for the system studied through high-resolution CCD spectroscopy. Again, knowing just the mass to arbitrarily high accuracy is not really useful unless the radii (or $\log g$ ) and composition of the stars are also known to the highest accuracy currently possible. Attempts to draw conclusions on finer details in stellar models from broad mean relations for binaries presumed to have "Population I" metallicity and without detailed, simultaneous fits to both components in each system (e.g. Stothers \& Chin 1991) are bound to remain inconclusive. 


\section{Conclusions}

Unquestionably, many areas of astronomy will benefit from yet another quantum leap in the quality and quantity of astrometric data available for stars to ever greater distances from us. The determination and astrophysical application of accurate binary masses can be one of these areas provided the intended applications are kept clearly in mind in both selecting the systems to be studied, carrying out the observations, and securing the additional data which, as discussed above, are crucial for the masses themselves to be really useful. As always, the best results in each case will be obtained through a judicious combination of the available astrometric, spectroscopic, and photometric techniques.

\section{References}

Andersen J., 1991, Astron. Astrophys. Rev. 3, 91

Andersen J., Clausen J.V., Magain P., 1989, Astron. Astrophys. 211, 346

Nordström B., Johansen K.T., 1994, Astron. Astrophys., in press

Popper D.M., 1980, Ann. Rev. Astron. Astrophys. 18, 115

Stothers R.B., Chin C.-W., 1991, Ap.J. 381, L67 